Note: this is the first online version of the final published paper - so prior to allocation of volume/issue/page numbers.

Citation (for the definitive version): $\mathrm{P}$ Newton, $\mathrm{R}$ Reeves, $\mathrm{E}$ West and $\mathrm{P}$ Schofield (2014). Patient-centred assessment and management of pain for older adults with dementia in care home and acute settings. Reviews in Clinical Gerontology, 24, pp 139-144. doi:10.1017/S0959259814000057.

Definitive version is available: http://dx.doi.org/10.1017/S0959259814000057 Copyright (C) Cambridge University Press 2014. 


\section{Reviews in Clinical Gerontology}

http://journals.cambridge.org/RCG

Additional services for Reviews in Clinical Gerontology:

Email alerts: Click here

Subscriptions: Click here

Commercial reprints: Click here

Terms of use : Click here

\section{Patient-centred assessment and management of pain for older adults with dementia in care home and acute settings}

P Newton, R Reeves, E West and P Schofield

Reviews in Clinical Gerontology / FirstView Article / February 2014, pp 1 - 6

DOI: 10.1017/S0959259814000057, Published online: 20 February 2014

Link to this article: http://journals.cambridge.org/abstract_S0959259814000057

How to cite this article:

P Newton, R Reeves, E West and P Schofield Patient-centred assessment and management of pain for older adults with dementia in care home and acute settings . Reviews in Clinical Gerontology, Available on CJO 2014 doi:10.1017/S0959259814000057

Request Permissions : $\underline{\text { Click here }}$ 


\title{
Patient-centred assessment and management of pain for older adults with dementia in care home and acute settings
}

\author{
P Newton, R Reeves, E West and P Schofield \\ Centre for Applied Health Research, University of Greenwich, London, UK
}

\begin{abstract}
Summary
Evidence suggests that there is a greater prevalence of pain, particularly chronic pain, in the older than in the younger population. This review looks at how dementia affects older people's ability to report pain, and indicates that pain is poorly assessed and managed in people living with dementia, in particular in care and acute settings. The review also reports findings from two recent studies looking at ways of improving the assessment and management of pain in acute settings. Multi-dimensional, patient-centred approaches to assessing and managing pain in those living with dementia are required, and future research should focus on innovative and practical approaches that can be applied in care home and acute settings.
\end{abstract}

Key words: ageing, pain, dementia, hospitals, care homes.

\section{Background}

Data suggest that there are approximately 700,000 people currently living with dementia in the UK, and this is expected to double to 1.4 million in the next 30 years, with a predicted cost of over $£ 50$ billion per year. ${ }^{1}$ The number of people living with dementia who will require care is set to increase; diagnosis is patchy and often delayed and carers need help and support to meet the challenges of looking after people with dementia. In recognition of this, the Dementia Challenge, launched in 2013 in the UK, sets out programmes of work in three key areas, called respectively 'Driving Improvements in Health and Care', 'Dementia Friendly Communities' and 'Improving Dementia Research'. It follows on from the implementation of 'Living Well With

Address for correspondence: Paul Newton, Centre for Applied Health Research, Faculty of Education and Health, University of Greenwich, Avery Hill Road, London SE9 2UG, UK.

Email: P.D.Newton@gre.ac.uk
Dementia: a National Dementia Strategy' set out by the Department of Health in 2009. ${ }^{2}$ Furthermore, in the UK, the Quality Care Standard published by the National Institute of Health and Clinical Effectiveness in $2013^{3}$ sets targets for health care providers, educationalists and researchers to develop innovative ways of providing future dementia care. The present review searched for literature relating to ageing, pain, dementia, acute settings and nursing/care homes through a comprehensive review of all databases. Fuller details of the search methods are available from the corresponding author.

Psycho-sociocultural factors may be important in the management of any individual experiencing pain, regardless of their age, and this requires a multidimensional person-centred perspective. Person-centred care emphasizes the psychological and social consequences of increasing mental frailty ${ }^{4}$ and acknowledges that many people with dementia can still carry out many of their normal daily activities independently. It focuses on helping people with dementia to maintain a sense of their own identity and wellbeing. ${ }^{5}$ The essence of person-centred care is to provide supportive and positive interactions to enable the person with dementia to feel valued and included. ${ }^{6}$ It includes valuing people, treating them as individuals, looking at the world from their perspective and understanding the importance of supportive social environments to provide continued opportunities to connect and communicate.

Older adults with dementia gradually lose their ability to communicate and process information, so they are less likely to express their pain in ways that are recognized by health care staff as typical ways of expressing pain. Instead, their manifestations of pain, which may include wandering, aggression and agitation are often seen as disruptive, problematic or challenging behaviour. $^{7}$ 
Pain and age: the incidence and management of chronic pain in older adults

Chronic pain is more prevalent in older than in younger adults. Recent research has found that the incidence of pain increases to $80 \%$ among older adults living in care homes. ${ }^{9}$ Some suggest that older people have an altered physiology of peripheral and central pain mechanisms and this, combined with psychological attitudes such as stoicism and reluctance to confirm the presence of pain means that older people may have different experiences of pain. ${ }^{8}$ In fact, there is only modest evidence of an age-related decline in pain-related sensitivity and the evidence is inconsistent. ${ }^{10}$ Others take the view that pain is present in the same format irrespective of age. ${ }^{11}$

A number of published articles have indicated that, and offered explanations of why, pain is poorly managed in older adults compared with their younger counterparts. Unfortunately there is a lack of evidence regarding acceptable treatment for older adults because trials tend to focus upon younger adults, ${ }^{12}$ and it is acknowledged that older adults are under-represented in pain clinics and pain management programmes. ${ }^{13}$ Other reasons cited include widespread assumptions among health care professionals that pain is an acceptable part of ageing, and older adults' assumptions that health care professionals will 'know' when they are in pain. ${ }^{12}$ The increased likelihood of co-morbidities associated with ageing can also mean that multiple medications may interact and cause unpleasant side-effects, thus adversely affecting patients' concordance with prescribed pain medication. Cognitive impairment associated with ageing may also make diagnosis more difficult.

\section{Pain in older adults with dementia who live in care home settings}

Husebo and colleagues demonstrated that people with dementia in nursing home care often have painful conditions that are poorly identified and managed. ${ }^{14} \mathrm{~A}$ qualitative study that sought to identify some of the barriers to reporting pain through a series of semi-structured interviews, conducted with residents living in a number of care homes within one district with mild to moderate cognitive impairment, identified a number of explanatory themes as follows:
A reluctance to report painlacceptance that pain is normal, and low expectations of help from medical interventions

Many residents when interviewed were in pain, but when asked why they had not reported the pain to the staff, they commented that there was no need to, as there was probably nothing that anyone could do.

\section{Fear of chemical/pharmacological interventions}

Many of the residents commented that they were fearful of using pharmacological interventions. They said they would prefer to manage without, or that nothing seemed to help.

\section{Age-related perceptions of pain}

Not only were the older age group (>80 years) reluctant to take analgesics, but they were also reluctant to admit that they had pain. Residents under 75 years were more willing to voice their pain, and to take analgesic drugs.

\section{Lack of awareness of potential pain relieving strategies}

The interviewees appeared keen to be involved in their own pain management. This was reflected in their comments of taking a hot bath or shower when the pain was bad, or 'rubbing the affected area'. However, they were often unaware of other approaches to pain self-management, such as relaxation, massage or distraction.

This research also highlighted other barriers to adequate pain management in older adults, related specifically to the attitudes of the staff, which are shown in Table 1. These have also been reported by Vallarand et al. ${ }^{16}$

These misconceptions may result in underprescribing, under-administration, delaying and withholding of analgesia and a failure to think beyond the traditional institutional practices. ${ }^{15,16}$ In addition, it is estimated that between 22 and $60 \%$ of older adults living in care homes have cognitive impairment. This can significantly diminish their ability to report pain and the carers' ability to identify pain. ${ }^{17}$ Due to a decline in ability to describe the problem verbally, adults with dementia will probably express their pain in 
Table 1. Beliefs held by staff in care homes which are a barrier to adequate pain management

- Belief that older people cannot tolerate opioid medications

- Persistent use of traditional analgesic methods as opposed to using some of the newer drugs

- A belief that not expressing pain means an absence of pain

- A belief that pain perception decreases with age

ways that are quite different from their cognitively intact counterparts, which can result in inadequate pain assessment and consequently poor pain management. ${ }^{18}$

The processing of sensory-discriminative aspects of pain occurs in the lateral pain system, whereas motivational-affective aspects are processed by the medial system. Experimental studies have shown the importance of the recognition of these two systems when dealing with patients with dementia. Scherder et al. demonstrated that pain thresholds (which are the sensory-discriminative aspects) did not differ between patients with Alzheimer's and those older adults without dementia, although pain tolerance (motivationalaffective aspect) did. ${ }^{19}$ This would suggest that older adults with Alzheimer's disease perceive the presence of pain, but that the intensity and affective aspects are different from that experienced by their cognitively intact counterparts, which might at least partially explain the atypical behavioural responses observed in this group. However, the research to date has been carried out with experimental pain and results may not be directly transferrable to adults in care homes, most of whom have chronic pain.

\section{Pain education in acute care}

Many adults in care settings have advanced forms of dementia ${ }^{17}$ and also have associated behavioural and communication difficulties associated with pain, ${ }^{7}$ which staff must manage on a day-to-day basis. In order to meet the challenge of providing supportive and positive patient-centred care to enable the person with dementia to feel valued and included in care settings, staff and carers need to understand how pain is experienced and reported by people living with dementia and how to manage it.

A recent pilot study by Schofield and Newton explored the knowledge, skills and attitudes of nursing staff working in an acute setting, towards identifying and managing pain in people living with dementia. The study began by developing a free to access, self-directed training package called 'The Assessment and Management of Pain and Dementia in Older Adults' (2012) aimed at healthcare professionals treating and caring for older adults living with dementia (available at: https://slondonhiec.org.uk/assessment-andmanagement-pain-older-adults-dementia). Participants were asked to fill out a validated questionnaire pre- and 1 week post-training, which gauged their knowledge, attitudes and skills in assessing and managing pain in people living with dementia. Baseline findings are presented in detail in Table 2. Senior nurses were more likely to report dementia patients as difficult to work with, and less qualified staff were more likely to report caring for people with dementia as unsatisfying. This illustrates the challenges that need to be taken into consideration when attempting to address how staff assess and manage pain and dementia in acute settings. The observation that having accurate knowledge of pain management in older adults with dementia was positively related to having previously taken part in dementia-related training indicates, however, that improvements can result from training, although more research is required to achieve a robust analysis of effective training to improve skills, knowledge and attitudes.

Senior managers within the acute setting who assisted with implementing the research suggested that, although participants were interested and committed to completing the training, nursing staff often found it difficult to make time to finish it. This may have been due to the length of the training package (87 pages of self-directed learning to be completed over 6 weeks). Given the low levels of previous training and existing knowledge, alongside the poor completion rates amongst the staff in this acute setting, the study also points to further challenges organizations face in seeking to improve the health and care of people living with dementia. Managers need to consider how 
Table 2. Baseline skills knowledge and attitudes of nursing staff in acute care setting

\begin{tabular}{ll}
\hline \hline Had received no dementia-related training during their employment & $58 \%$ \\
Disagreed that there was little they could do to help people living with dementia & $84 \%$ \\
Did not enjoy working with patients with dementia & $17.5 \%$ \\
Found older adults with dementia difficult to work with & $26 \%$ \\
Believed adults with dementia do not experience pain & $10 \%$ \\
Believed they could identify pain in older adults with dementia & $73 \%$ \\
Knew of pain assessment tools for adults with dementia & $34 \%$ \\
\hline
\end{tabular}

organizational resources can be targeted to ensure that the dementia and pain-related training needs of staff are identified and met, as well as assessing the most appropriate ways of delivering this type of training.

\section{Pain in acute hospital settings: the feedback study}

Raising awareness among ward staff of their own patients' experiences of pain could help to improve pain management in hospitals. Members of our team recently conducted a 2-year intervention study to assess the impact of targeted patient feedback on subsequent patients' experiences in the two acute National Health Service (NHS) hospitals. ${ }^{20}$ Wards were randomly allocated to one of three groups:

1. Basic Feedback, where nurses were sent a written report detailing the results of the patient survey including descriptive statistics and qualitative comments.

2. Feedback Plus, where, in addition to receiving the same style of written reports, nurses also attended meetings with researchers to discuss the results and plan improvements.

3. Control, where data about patients experiences were collected but no special efforts were made to communicate them to ward staff.

Further information about the study design can be accessed at: http://www.biomedcentral. com/content/pdf/1472-6963-13-259.pdf.

To measure patients' experiences of ward nursing care the National Inpatient Survey questionnaire was used, which includes a question about pain: 'Did you think hospital staff did everything possible to control your pain?', for which there are three response options: 'Yes, definitely', 'Yes, to some extent' or 'No'. (Those who said they did not experience pain while in hospital are excluded with a preceding filter question.) Over the course of the study period, in postal surveys of recently discharged inpatients, a total of 2629 responses to the pain question were returned.

The study's main finding was that written feedback ('Basic Feedback') alone had no effect, even when ward-specific results were presented, but supplementing the written feedback with ward meetings ('Feedback Plus') had a highly significant positive effect on patients' experiences. While these findings may be important for understanding pain management in acute hospital wards, it is important to emphasize however, that this study was not designed specifically for the purpose of investigating patients' experiences of pain and before the study was conducted, we did not have any specific hypotheses relating to pain.

Separately for each participating hospital, comparing baseline (beginning of the study) and final (end of study) results, we assessed the percentage of patients who gave the most positive response to whether staff did everything possible to control their pain. In Hospital A, the percentage of patients responding 'Yes, definitely' increased by $7 \%$ in Feedback Plus wards, declined by $3 \%$ in Basic Feedback wards and increased by $13 \%$ in Control wards, providing equivocal evidence for the impact of Feedback Plus. In Hospital $\mathrm{B}$, the percentage of patients who responded positively to the pain question increased by $2 \%$ in Feedback Plus wards, declined by $27 \%$ in Basic Feedback wards and declined by $15 \%$ in Control wards, suggesting that Feedback Plus had a positive impact on patients' experiences of pain by counteracting a general decline in patients' experiences in Hospital B, possibly benefitting a substantial number of patients as a result of the targeted feedback and facilitated meetings with ward nursing staff. However, good evidence in this area will require the design and implementation of a complex intervention study focusing specifically on pain and adapted for use with patients with dementia. 
Pain in acute hospitals: age differences among older adults

To date, little attention has been given to differences among those aged over the national retirement age in their experiences of acute hospital care. The archived data from the National Inpatient Survey (Care Quality Commission, 2012), on which the feedback study described above was based, provides patients' age information in only four categories, the oldest of which is ' $66+$ ', which accounts for more than half $(55 \%)$ of responders. It is widely understood that perception of their care improves as the patient's age increases, and this is thought to be an age-related reporting bias. ${ }^{2}$ To allow for age differences among NHS trusts, results generated by the National In-patient Surveys are age-adjusted to control for this bias.

The data from the Feedback study provide more detailed information on patients' ages and allow us to divide the older age group into those aged 66-80 years and those aged 81 and older. In each age category, the percentage of patients who said staff 'definitely' did everything possible to control their pain was as follows: age $16-35$ years: $57 \%$; age $36-50$ years: $67 \%$; age $51-65$ years: $69 \%$; age 68-80 years: $71 \%$; age 81 years or older: $65 \%$. These results show a steady improvement in pain care experiences as patients' ages increase until they reach the oldest age category, when it declines. This suggests that further investigation of older adults' experience of pain in acute hospitals is warranted, and that distinguishing different age groups among older people could provide important evidence that would be missed if older people were treated as one group.

\section{Conclusion}

Research and policy drivers highlight that healthcare professionals need to adopt and enhance patient-centred approaches to dementia to ensure the best quality care is available for the increasing numbers of people living with dementia. Despite the fact that both chronic and acute pain are increasingly reported by older people, it is often poorly managed, particularly in older people living with dementia. Studies have shown that this is not due solely to a general stoicism and underreporting by older adults. Older people with a cognitive impairment, such as dementia, may be unable to express their pain, or may express their pain in ways that are seen as challenging and disruptive within care and acute environments. Although knowledge of the potential affective and behavioural manifestations of living with pain and dementia provides us with some methods of assessment, it is clear that further research is required in this area, particularly in relation to experiences of chronic pain.

When people living with dementia are in care and acute settings they are often reliant upon the skills, knowledge and attitudes of staff to address their needs. It has been highlighted that staff often lack skills in managing pain in people living with dementia. Giving detailed feedback to staff about patients' experiences of pain can improve pain management and assessment. This feedback, combined with targeted pain and dementia training, could offer a practical, low-cost way of improving pain management for people living with dementia. Lack of organizational support for this type of training may mean, however, that staff lack the time and opportunity to enhance their skills. More research is required into the experiences of those aged 80 and over, as this group is particularly vulnerable to experiencing pain in acute settings, and more likely to access these settings. If, as has been suggested, a multi-dimensional perspective that centres on the individual is needed, then further innovative research to elucidate effective components of this is also required.

\section{Financial support}

The study of nurses' skills, knowledge and attitudes to the assessment and management of pain and dementia in acute settings was supported by NHS South East London Health Innovation and Education Cluster. The 'Feedback Study' was supported by a University of Greenwich Research and Enterprise grant.

\section{Conflicts of interest}

None

\section{References}

1 de Waal H, Lyketsos C, Ames D, O’Brien J. Designing and Delivering Dementia Services. Available at: http://onlinelibrary.wiley. 
com/book/10.1002/9781118378663 (accessed 11 November 2013).

2 Department of Health (2009) 'Living Well With Dementia: a national dementia strategy'. Available at: https://www.gov.uk/government/ uploads/system/uploads/attachment_data/file/ 168220/dh_094051.pdf (accessed 28 October 2013).

3 National Institute of Health and Clinical Effectiveness (2013) Mental wellbeing of older people in residential care: quality standard consultation. Available at: http://www.nice.org. $\mathrm{uk} /$ guidance/index.jsp? action $=$ folder $\& \mathrm{o}=64372$ (accessed 28 October 2013).

4 Kitwood T. The experience of dementia. Aging Mental Health 1997, 1: 13-22.

5 Sabat S. The Experience of Alzheimer's Disease: Life Through a Tangled Veil. 2001; Chicester: Wiley.

6 Brooker D. What is person-centred care in dementia? Rev Clin Gerontol 2003, 13: 215-22.

7 Hyochol A, Horgas L. The relationship between pain and disruptive behaviors in nursing home resident with dementia. BMC Geriatrics 2013, 13: 13-14.

8 Pickering G, Jourdan D, Dubray C. Acute versus chronic pain treatment in Alzheimer's disease. Eur J Pain 2006, 10: 379-84.

9 Elliott A. Prevalence of pain in older adults. Age Ageing 2013, 42: 1.

10 Edwards RR. In Gibson SJ, Weiner DK (eds), Pain in plder persons, progress. Pain Research and Management 35, pp. 45-65. Seattle: IASP Press, 2005.

11 Sorkin BA, Rudy TE, Hanlon RB, Turk DC, Stieg RL. Chronic pain in old and young patients: differences appear less important than similarities. J Gerontol 1990; 45: 64-68.
12 Gibson SJ. Older people's pain. Pain: Clinical Updates 2006, 14: 1-4.

13 Kee WG, Middaugh SJ, Redpath S, Hargadon R. Age as a factor in admission to chronic pain rehabilitation. Clin J Pain 1998; 14: 121-28.

14 Husebo BS, Strand LI, Moe-Nilssen R, Borgehusebo S, Aarsland D, Ljunggren AE. Who suffers most? Dementia and pain in nursing home patients: a cross-sectional study. J Am Med Dir Assoc 2008, 9: 427-33.

15 Schofield PA. Talking to older people in care homes: Perceptions of their pain and their preferred management strategies. Results of a pilot study. Int J Disability Human Dev 2006, 5: 53-59.

16 Vallerand AH, Hasenau SM, Templin T. Barriers to pain management by home care nurses.

Home Healthc Nurse 2004, 22:

831-38.

17 Abbey J, Piller N, De Bellis A, Esterman A, Parker D, Giles L, Lowcay B. The Abbey pain scale: a 1-minute numerical indicator for people with end-stage dementia. Int J Palliat Nurs 2004, 10: 6-13.

18 Simons W, Malabar R. Assessing pain in elderly patients who cannot respond verbally. J Adv Nurs 1995, 22: 663-69.

19 Scherder EJ, Oosterman JM, Ooms ME, Ribbe MW, Swaab DF. Chronic pain in dementia and in disorders with a high risk for congnitive impairment. Tijdschr Gerontol Geriatr 2005, 36: 116-21.

20 Reeves R, West E, Barron D. Facilitated patient experience feedback can improve nursing care: a pilot study for a phase III cluster randomised controlled trial. BMC Health Services Res 2013: 259. 